\title{
BMJ Open Study protocol for a randomised controlled trial on the effect of local analgesia for pain relief after minimal invasive sacroiliac joint fusion: the ARTEMIS study
}

Sem M M Hermans (D) , ${ }^{1}$ Jorm M Nellensteijn, ${ }^{2}$ Henk van Santbrink, ${ }^{3,4}$ Rob Knoef, ${ }^{2}$ Mattheus K Reinders, ${ }^{5}$ Daisy M N Hoofwijk, ${ }^{6}$ Jan W Potters, ${ }^{7}$ Kris L L Movig, ${ }^{8}$ Inez Curfs, ${ }^{1}$ Wouter L W van Hemert ${ }^{1}$

To cite: Hermans SMM, Nellensteijn JM, van Santbrink H, et al. Study protocol for a randomised controlled trial on the effect of local analgesia for pain relief after minimal invasive sacroiliac joint fusion: the ARTEMIS study. BMJ Open 2021;11:e056204. doi:10.1136/ bmjopen-2021-056204

- Prepublication history and additional supplemental material for this paper are available online. To view these files, please visit the journal online (http://dx.doi.org/10.1136/ bmjopen-2021-056204)

Received 05 August 2021 Accepted 30 November 2021

Check for updates

(c) Author(s) (or their employer(s)) 2021. Re-use permitted under CC BY-NC. No commercial re-use. See rights and permissions. Published by BMJ.

For numbered affiliations see end of article.

Correspondence to

Sem M M Hermans;

semhermans@hotmail.com

\section{ABSTRACT}

Introduction Chronic lower back pain is a common report in the general population. A dysfunctional sacroiliac joint (SIJ) is estimated to be responsible for one in five patients with lower back pain. Minimally invasive sacroiliac joint fusion (MISJF) is a surgical procedure to treat SIJ dysfunction. During the procedure, the SIJ is stabilised by implants inserted percutaneously under fluoroscopy guidance. Postoperatively, patients often report a lot of pain, which contributes to patients taking high doses of painkillers (opioids for example,) and preventing early mobilisation. In several orthopaedic procedures, intraoperative infiltration of the wound bed results in decreased consumption of analgesics, earlier mobilisation and shorter hospitalisation time. The aim of this study is to investigate the effectiveness of intraoperative SIJ infiltration with analgesia in reducing postoperative pain after MISJF. Methods and analysis We will perform a two-centre, prospective, double-blind, randomised controlled trial to determine whether SIJ infiltration with 1.5-5cc bupivacaine $0.50 \%$ is superior to $1.5-5 \mathrm{cc}$ placebo $(\mathrm{NaCl}$ $0.9 \%$ ) in reducing postoperative pain in patients after MISJF, and to determine whether bupivacaine significantly reduces opioid use in the direct postoperative period. Patients will be randomised with 1:1 allocation for either bupivacaine (intervention) or placebo SIJ infiltration. Postoperative pain will be measured by the Visual Analogue Scale pain score at entry and exit recovery, 2, 4, 6,24 and 48 hours postoperatively.

Ethics and dissemination This is the first trial that investigates the effectiveness of intraoperative SIJ infiltration with bupivacaine $0.50 \%$ in reducing postoperative pain after MISJF. If intraoperative SIJ infiltration with bupivacaine $0.50 \%$ proves to be effective, this might have important clinical implications, such as postoperative analgesics (opioids for example,) consumption, earlier mobilisation and potentially shorter hospitalisation time.

Trial registration number NL9151.

\section{INTRODUCTION}

Chronic lower back pain is a common report in the general population. A dysfunctional
Strengths and limitations of this study

- The proposed study design, a double-blinded, placebo-controlled, randomised trial, is the best available method to investigate the effectiveness of intraoperative sacroiliac joint (SIJ) infiltration with bupivacaine $0.50 \%$ in reducing postoperative pain after minimally invasive sacroiliac joint fusion (MISJF).

- It is a multicentre study, involving two high-volume MISJF centres in the Netherlands, which increases the generalisability of the results.

- Infiltrating the SIJ under fluoroscopy guidance at the end of the procedure is a simple, reproducible method to deliver an intra-articular bolus of analgesia.

- Although the primary outcome is a validated tool, the Visual Analogue Scale (VAS) pain score remains a patient-reported outcome measure (PROM) and is thereby at risk for some sort of subjective discrepancies.

sacroiliac joint (SIJ) is estimated to be responsible for one in five patients with lower back pain. ${ }^{12}$ Surgical intervention for SIJ dysfunction is considered if pain is refractory to conservative treatment options. Minimally invasive sacroiliac joint fusion (MISJF) is the most common surgical procedure to treat chronic low back pain due to SIJ dysfunction. During the procedure, the SIJ is stabilised by implants inserted percutaneously under intraoperative fluoroscopy guidance. ${ }^{3}$ Postoperatively, patients often report a lot of pain, which contributes to patients taking high doses of painkillers and preventing early mobilisation. Painkillers, especially opioids, can cause nausea and drowsiness, resulting in a prolonged hospitalisation period. ${ }^{4}$ Postoperative pain and nausea are also a major 
cause of a negative experience of hospitalisation. ${ }^{5}$ In several orthopaedic procedures, intraoperative infiltration of the wound bed results in decreased consumption of analgesics, earlier mobilisation and shorter hospitalisation time. ${ }^{6-8}$

As the implants are inserted laterally through the gluteal musculature during MISJF, and the SIJ is punctured to place the implants, pain is expected on loading the operated side. In the first 3 weeks after surgery patients mobilise with crutches, as $50 \%$ weight-bearing is allowed. ${ }^{9}$ Like most other orthopaedic procedures, early postoperative mobilisation aids in the process of recovery, as this can prevent; fear of movement, prolonged hospitalisation, thrombosis and possibly chronic pain reports. ${ }^{10}$ Irrigation of the incision with bupivacaine before closure of the wound is often performed in MISJF. Lately, some surgeons also perform an intra-articular SIJ infiltration at the end of the procedure to diminish postoperative pain and promote early mobilisation, however the effects of such an infiltration are unclear and have never been described in scientific literature. One can postulate the potential benefits of delivering local analgesia in the SIJ. Furthermore, infiltrating the SIJ only takes a few extra minutes of operating time and a minimal amount of fluoroscopy screening time. The aim of this study is to determine whether intraoperative intra-articular analgesia with bupivacaine $0.50 \%$ is superior to placebo (intraoperative intra-articular infiltration of $\mathrm{NaCl} 0.9 \%$ ) in reducing postoperative pain in patients after MISJF, and to determine whether opioid use in the first 48 hours after surgery is significantly higher in the placebo group.

\section{METHODS AND ANALYSIS}

\section{Study design}

This is a prospective, double-blind, randomised controlled trial (blinding for the patient, clinician, researcher and statistician) that investigates the effectiveness of intraoperative SIJ infiltration with bupivacaine $0.50 \%$ in reducing postoperative pain after MISJF. Patients will either receive $1.5-5 \mathrm{cc}$ bupivacaine $0.50 \%$ or $1.5-5 \mathrm{cc}$ placebo $(\mathrm{NaCl}$ $0.9 \%$ ) intraoperatively. Treatment is always by dedicated spine or pelvic surgeons, who have experience with infiltrating the SIJ. A flowchart detailing the study design is outlined in figure 1.

\section{Patient and public involvements \\ No patient involved.}

\section{Participants and recruitment}

Adult patients referred to the orthopaedic outpatient clinic who are candidates for MISJF surgery are potentially eligible to participate in this study. An indication for MISJF is based on medical interviewing, medical examination including the following SIJ provocative tests; flexion abduction external rotation (FABER test), thigh thrust, Gaenslen's test, sacral distraction, lateral compression and sacral thrust and an image-guided intra-articular SIJ injection with local anaesthetic according to a specific guideline. ${ }^{11} 12$ At least three of five provocative tests should evoke SIJ pain and at least a 50\% reduction of SIJ pain 30-60 min following image-guided injection should occur to be eligible for MISJF. In order to be eligible to participate in this study, a subject must meet all of the following criteria:
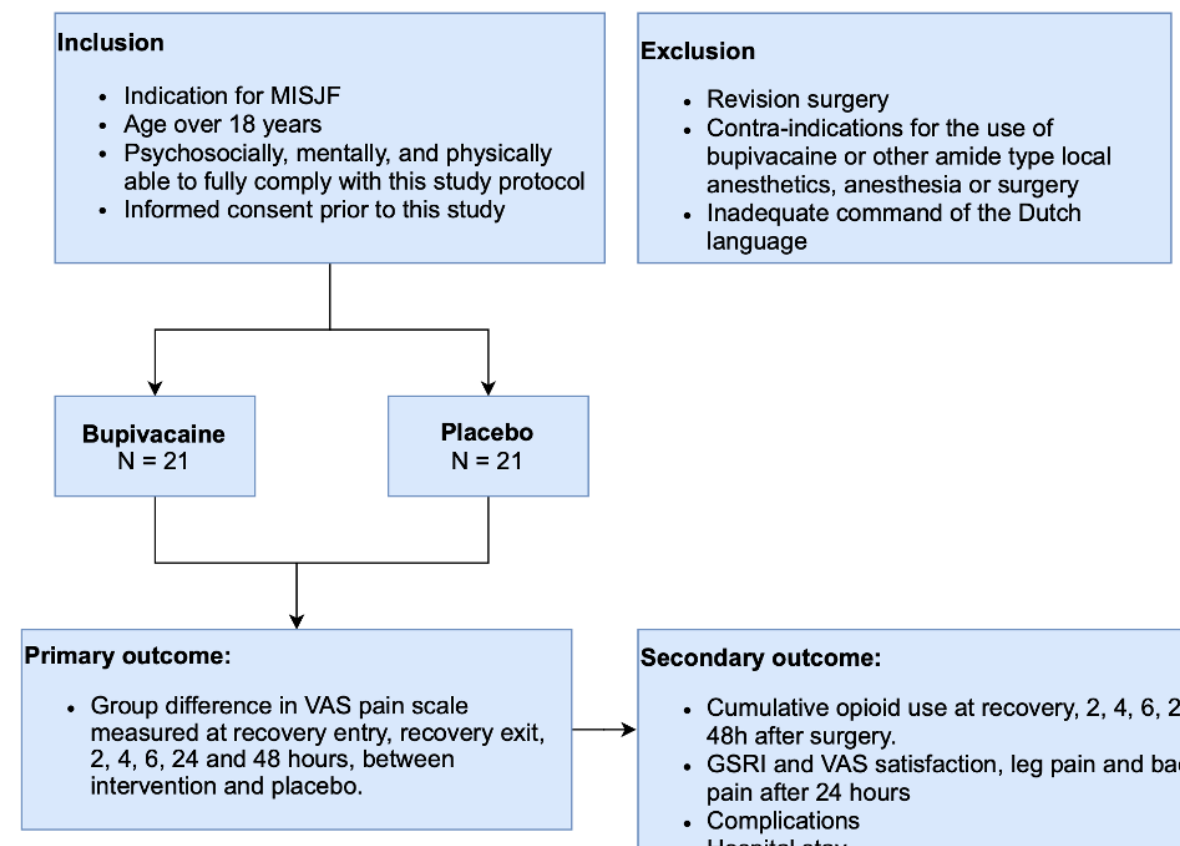

- Revision surgery

Contra-indications for the use of

Inadequate command of the Dutch

language

Figure 1 Flowchart of study design. GSRI, General Surgery Recovery Index; MISJF, minimally invasive sacroiliac joint fusion; VAS, Visual Analogue Scale. 
1. Indication for MISJF surgery.

2. Age over 18 years.

3. Psychosocially, mentally and physically able to fully comply with this study protocol.

4. Informed consent prior to this study.

A potential subject who meets any of the following criteria will be excluded from participation in this study:

1. Revision surgery.

2. Contra-indications for the use of bupivacaine or other amide type local anaesthetics, anaesthesia or surgery.

3. Inadequate command of the Dutch language.

Patients eligible for inclusion will be referred to the researchers. The researchers will inform the patient, and when they are willing to participate, include them. A copy of the patient consent form can be found in the appendix as (online supplemental item 1 ).

\section{Outcome measures}

The primary outcome is the difference in VAS pain between intervention and placebo groups during the first 48 hours after surgery, with interval measurements at recovery entry, recovery exit, 2, 4, 6, 24 and 48 hours. The following secondary outcome measures will be evaluated:

- The cumulative postoperative opioid consumption.

- Patient satisfaction measured using General Surgery Recovery Index (GSRI) and Visual Analogue Scale (VAS) satisfaction. Patients will fill out the questionnaire 24 hours after surgery. In addition, VAS leg pain and back pain will be filled out 24 hours after surgery.

- Adverse events; postoperative infection, deep venous thrombosis, haematoma, neurological deficits and other complications as pneumonia, urine retention or urinary tract infection. Adverse events will be followed up to 30 days.

- Hospital stay defined as days spent in hospital after surgery.

Other study parameters are sex, age, body mass index (BMI), preoperative opioid usage, occurrence of diabetes, diagnosis, previous pelvic or back surgery, preoperative VAS pain and American Society of Anesthesiologists (ASA) classification. The amount of fluid that the surgeon is able to infiltrate in the SIJ, duration of surgery, intraoperative blood loss and intraoperative opioid administration will be monitored as well.

\section{Randomisation}

Allocation is performed by the pharmacist, blinded for clinicians, researchers, patients and statisticians. The pharmacy will prepare blinded syringes with either bupivacaine $0.50 \%$ or $\mathrm{NaCl}$. The pharmacy will mark the syringes with a kit number (1, 2, 3 and so on). These numbers will correspond with a computer-generated randomisation list which will be stored by the pharmacy. The researchers need to order the syringes when the surgery is planned. They will be collected on the day of surgery before a study patient will be operated on. Once they leave the pharmacy the syringes can be kept for 24 hours at room temperature. If a patient is eligible for randomisation the successive syringe will be used. The surgeon will note the kit number in the patient's electronic dossier. Once the study is completed the randomisation list will be unblinded by the pharmacy to the clinicians, researchers, statisticians and patients (if desired).

\section{Sample size calculation}

Difference in pain between SIJ infiltration with $1.5-5 \mathrm{cc}$ bupivacaine $0.50 \%$ and placebo is the primary endpoint and was used to calculate the sample size. Based on our own data from a pilot study, derived from recovery unit charts, we estimated that the SD of the pain score will be about 2.2. A two-point reduction on the 11-points ( 0 to 10) VAS pain score is considered clinically relevant. ${ }^{13} 14$ In order to obtain a clinically meaningful effect with $80 \%$ power, 19 patients are required per group. Because no contrast is implemented during infiltration, there is an estimated chance of $10 \%$ that the infiltration will not be administered intra-articular but periarticular. Although the analgesic effect of intra-articular and periarticular infiltration is similar, this has been taken into account in the sample size calculation. ${ }^{15}{ }^{16}$ Subsequently, 42 patients (21 patients per group) should be enrolled in this study.

\section{Statistical analysis}

Frequency tables will be provided for all categorical demographic information. Continuous variables will be presented as mean $\pm \mathrm{SD}$ or median $\pm \mathrm{IQR}$ depending on the distribution of the data. Analysis will be performed by principal investigators using IBM SPSS statistical software package V.27 (SPSS). Missing values will be imputed using stochastic regression imputation using full conditional specification.

The primary outcome is the group difference in VAS pain score on arrival and exit recovery, 2, 4, 6, 24 and 48 hours. The difference will be tested using the independent-samples t-test. In addition, we will determine the group differences over time (ie, the slopes of the relation between time and pain) using a linear mixedeffects model with a random intercept and slope of time. The model will include group and time as covariates, and the interaction between group and time.

The secondary outcome measures will be determined as followed:

- The difference in cumulative opioid use during stay at recovery, 24 hours and 48 hours after surgery will be analysed using a linear mixed-effects model.

- Patient satisfaction measured using GSRI and VAS.

- Analysis of these patient-reported outcome measures will be achieved using a linear mixed-effects model.

- The proportion and kind of postoperative complications will be compared by means of logistic regression analysis.

- Difference in length of hospital stay defined as days spent in hospital after surgery will be assessed by linear regression or Poisson regression, depending on the distribution of hospital stay. 
Other study parameters as descriptive statistics (sex, age, BMI for example,) will be calculated.

\section{Treatment of subjects}

MISJF will be performed as standard care. All patients receive general anaesthesia, are intubated and then positioned in prone position. Short-acting opioids like sufentanil or fentanyl will be used during surgery; however, morphine will not be administered at the end of surgery as a base for postoperative pain relief. After anaesthesia is administered the patient is prepped in sterile fashion. Intraoperative fluoroscopy is used during surgery for optimal placement of implants. Lateral view and pelvic inlet and outlet views are used to obtain an appropriate starting point. A $3 \mathrm{~cm}$ lateral incision is made across the sacral midline. A guide pin is placed across the ilium and across the SIJ. A drill is used to create a pathway and decorticate the bone. A triangular broach is then used to further decorticate the bone and prepare the pathway to receive the first implant. This implant is mostly seated within the sacral ala. The second implant is generally located above or adjacent to the $\mathrm{S} 1$ foramen and the third between the $\mathrm{S} 1$ and $\mathrm{S} 2$ foramen.

After closure of the incision a spinal needle is used to infiltrate the SIJ (intra-articular) under fluoroscopy guidance. Either bupivacaine $0.50 \% 1.5-5 \mathrm{cc}$ (intervention) or $\mathrm{NaCl} 0.9 \%$ 1.5-5 cc (placebo) will be infiltrated. Bupivacaine $0.50 \%$ is chosen for the intervention group as it has proven to be effective in reducing pain in patients suffering from SIJ dysfunction. ${ }^{17} 18$ Both groups receive the same perioperative protocol. This includes:

- Preoperative cefazolin (2 $\mathrm{g}$ intravenous, $30 \mathrm{~min}$ before incision or adequate alternative whenever a patient is allergic).

- For postoperative analgesia, all patients will be prescribed acetaminophen four times $1000 \mathrm{mg}$ daily either intravenous or oral.

- Standard physical therapy during hospitalisation for mobilisation instructions.

- Peripheral physical therapy starts 2 weeks postoperatively with for example, gluteal strengthen training.

- Deep venous thrombosis prophylaxis according to hospital protocol.

After surgery patients will be transported to the recovery room, where they will be monitored for a minimum time of 1 hour. During their stay at the recovery room and at the ward patient will receive intravenous or intramuscular piritramide until VAS pain $\leq 3$. Dosage is determined based on VAS pain score and body weight, $0.2-0.3 \mathrm{mg} / \mathrm{kg}$ with a maximum of $80 \mathrm{mg} /$ day in four dosages.

\section{Ethics and dissemination}

Ethical approval has been granted by the Medical Ethical Committee Zuyderland, Heerlen, the Netherlands. Informed consent will be obtained in writing from all participants prior to study enrolment. Study results will be disseminated through presentation at a peer-reviewed medical journal. We also plan to present our study results at selected conferences and scientific meetings.

\section{Trial status}

This study is in the process of recruiting participants as of January 2021, and it is expected that data regarding the intervention effects will be available at the end of 2023 .

\section{Author affiliations}

${ }^{1}$ Orthopaedic Surgery, Zuyderland Medical Centre Heerlen, Heerlen, Limburg, Netherlands

${ }^{2}$ Orthopaedic Surgery, Medical Spectrum Twente, Enschede, Netherlands ${ }^{3}$ Neurosurgery, Zuyderland Medical Centre Heerlen, Heerlen, Netherlands ${ }^{4}$ Neurosurgery, Maastricht Universitair Medisch Centrum+, Maastricht, Limburg, Netherlands

${ }^{5}$ Clinical Pharmacy, Pharmacology and Toxicology, Zuyderland Medical Centre Heerlen, Heerlen, Limburg, Netherlands

${ }^{6}$ Anaesthesiology, Zuyderland Medical Centre Heerlen, Heerlen, Limburg, Netherlands

${ }^{7}$ Anaesthesiology, Medical Spectrum Twente, Enschede, Netherlands

${ }^{8}$ Department of Clinical Pharmacy, Medical Spectrum Twente, Enschede, Netherlands

Contributors SMMH: concept and design, drafting protocol. RK: concept and design, drafting protocol. HvS: critical revision of protocol, supervision. JMN: concept and design, critical revision of protocol, supervision. MKR: critical revision of protocol, supervision. DMNH: critical revision of protocol, supervision. JWP: critical revision of protocol, supervision. KLLM: critical revision of protocol, supervision. WLWvH: concept and design, critical revision of protocol, supervision. IC: concept and design, critical revision of protocol, supervision.

Funding The authors have not declared a specific grant for this research from any funding agency in the public, commercial or not-for-profit sectors.

\section{Competing interests None declared.}

Patient consent for publication Not applicable.

Ethics approval This study involves human participants and was approved by METCZ20210069. Participants gave informed consent to participate in the study before taking part.

Provenance and peer review Not commissioned; externally peer reviewed.

Supplemental material This content has been supplied by the author(s). It has not been vetted by BMJ Publishing Group Limited (BMJ) and may not have been peer-reviewed. Any opinions or recommendations discussed are solely those of the author(s) and are not endorsed by BMJ. BMJ disclaims all liability and responsibility arising from any reliance placed on the content. Where the content includes any translated material, BMJ does not warrant the accuracy and reliability of the translations (including but not limited to local regulations, clinical guidelines, terminology, drug names and drug dosages), and is not responsible for any error and/or omissions arising from translation and adaptation or otherwise.

Open access This is an open access article distributed in accordance with the Creative Commons Attribution Non Commercial (CC BY-NC 4.0) license, which permits others to distribute, remix, adapt, build upon this work non-commercially, and license their derivative works on different terms, provided the original work is properly cited, appropriate credit is given, any changes made indicated, and the use is non-commercial. See: http://creativecommons.org/licenses/by-nc/4.0/.

ORCID iD

Sem M M Hermans http://orcid.org/0000-0002-9484-919X

\section{REFERENCES}

1 Bernard TN, Kirkaldy-Willis WH. Recognizing specific characteristics of nonspecific low back pain. Clin Orthop Relat Res 1987:266???280.

2 Sembrano JN, Polly DW. How often is low back pain not coming from the back? Spine 2009;34:E27-32.

3 Tran ZV, Ivashchenko A, Brooks L. Sacroiliac joint fusion methodology - minimally invasive compared to screw-type 
surgeries: A systematic review and meta-analysis. Pain Physician 2019;22:29-40.

4 Benyamin R, Trescot AM, Datta S, et al. Opioid complications and side effects. Pain Physician 2008;11:S105-20.

5 Gan TJ. Poorly controlled postoperative pain: prevalence, consequences, and prevention. J Pain Res 2017;10:2287-98.

6 Dysart SH, Barrington JW, Del Gaizo DJ, et al. Local infiltration analgesia with liposomal bupivacaine improves early outcomes after total knee arthroplasty: 24-hour data from the Pillar study. $J$ Arthroplasty 2019;34:882-6.

$7 \mathrm{Kim} \mathrm{H}-\mathrm{J}$, Ahn HS, Nam Y, et al. Comparative study of the efficacy of transdermal buprenorphine patches and prolonged-release tramadol tablets for postoperative pain control after spinal fusion surgery: a prospective, randomized controlled non-inferiority trial. Eur Spine $J$ 2017:26:2961-8.

8 Pace V, Gul A, Prakash V, et al. Wound infiltration with levobupivacaine, ketorolac, and adrenaline for postoperative pain control after spinal fusion surgery. Asian Spine J 2021;15:539-44.

9 Smith AG, Capobianco R, Cher D, et al. Open versus minimally invasive sacroiliac joint fusion: a multi-center comparison of perioperative measures and clinical outcomes. Ann Surg Innov Res 2013;7.

10 Kaye AD, Urman RD, Cornett EM, et al. Enhanced recovery pathways in orthopedic surgery. $J$ Anaesthesiol Clin Pharmacol 2019;35:S35-S39.
11 Society ISI,Bogduk N. Isis practice guidelines for spinal diagnostic and treatment procedures. 2nd edn. International Spine Intervention Society, 2014. https://books.google.nl/books?id=8w6NoAEACAAJ

12 Laslett M, Aprill CN, McDonald B, et al. Diagnosis of sacroiliac joint pain: validity of individual provocation tests and composites of tests. Man Ther 2005;10:207-18.

13 Salaffi F, Stancati A, Silvestri CA, et al. Minimal clinically important changes in chronic musculoskeletal pain intensity measured on a numerical rating scale. Eur J Pain 2004;8:283-91.

14 Myles PS, Myles DB, Galagher W, et al. Measuring acute postoperative pain using the visual analog scale: the minimal clinically important difference and patient acceptable symptom state. Br J Anaesth 2017;118:424-9.

15 Cohen SP, Chen Y, Neufeld NJ. Sacroiliac joint pain: a comprehensive review of epidemiology, diagnosis and treatment. Expert Rev Neurother 2013;13:99-116.

16 Simopoulos TT, Manchikanti L, Gupta S, et al. Systematic review of the diagnostic accuracy and therapeutic effectiveness of sacroiliac joint interventions. Pain Physician 2015;18:E713-56.

17 Kennedy DJ, Engel A, Kreiner DS, et al. Fluoroscopically guided diagnostic and therapeutic intra-articular Sacroiliac joint injections: a systematic review. Pain Med 2015;16:1500-18.

18 Scholten PM, Patel SI, Christos PJ, et al. Short-Term efficacy of sacroiliac joint corticosteroid injection based on arthrographic contrast patterns. Pm R 2015;7:385-91. 